\title{
Fruit and vegetable consumption and muscle strength and power during adolescence: the Northern Ireland Young Hearts Project
}

\author{
C. E. Neville ${ }^{1}$, M. C. McKinley ${ }^{1}$, L. J. Murray ${ }^{1}$, C. A. Boreham ${ }^{2}$, J. V. Woodside ${ }^{1}$ \\ and on behalf of the Young Hearts Study Group \\ ${ }^{1}$ Nutrition and Metabolism Research Group, Centre for Public Health, Queen's University Belfast, Belfast, BT12 6BJ, \\ UK and ${ }^{2}$ University College Dublin, Belfield, Dublin 4, Ireland
}

Epidemiological studies suggest that increased fruit and vegetable (FV) consumption may improve muscle strength and power. However, the evidence is inconclusive and the majority of studies have been conducted in older adults ${ }^{(1-4)}$. The current study examined the association between FV consumption and muscle strength and power in an adolescent population.

A cross-sectional analysis was carried out on data obtained from 1019 boys and 998 girls, aged 12 and 15 years, who participated in The Young Hearts 2000 Study. FV consumption was assessed by diet history. Grip strength (muscle strength) and vertical jump power (muscle power) was assessed with a dynamometer and Jump-MD meter, respectively. Associations between FV consumption and strength and power were assessed by regression modelling, with adjustment for potential confounders.

Boys and girls with high FV intakes ( $>237.71 \mathrm{~g} / \mathrm{d}$ and $>267.57 \mathrm{~g} / \mathrm{d}$, respectively, based on the highest third) had significantly higher jump power than those with low intakes, after adjustment for confounding factors. Girls with high FV intakes also had higher grip strength than those with low FV intake, although significance was lost after adjusting for physical activity. No significant associations were evident between FV intake and grip strength in boys after adjusting for confounding factors. Similar findings were observed when FV intakes were analysed separately.

\begin{tabular}{|c|c|c|c|c|}
\hline \multirow[b]{3}{*}{ FV Categories } & \multicolumn{2}{|l|}{ Grip strength $(\mathrm{kg})$} & \multicolumn{2}{|l|}{ Vertical jump power $(\mathrm{cm})$} \\
\hline & Boys & Girls & $\overline{\text { Boys }}$ & Girls \\
\hline & Difference in mean $(95 \% \mathrm{CI})$ & Difference in mean $(95 \% \mathrm{CI})$ & Difference in mean $(95 \% \mathrm{CI})$ & Difference in mean $(95 \% \mathrm{CI})$ \\
\hline \multicolumn{5}{|l|}{ Model 1} \\
\hline High intake $^{\mathrm{a}}$ & - & - & - & - \\
\hline Moderate intake & $-1 \cdot 56(-2 \cdot 94,-0 \cdot 18)^{*}$ & $-0.63(-1.41,0 \cdot 14)$ & $-1 \cdot 21(-2 \cdot 51,0 \cdot 10)$ & $-1 \cdot 14(-2 \cdot 08,-0 \cdot 19)^{*}$ \\
\hline Low intake & $-0 \cdot 58(-1 \cdot 96,0 \cdot 80)$ & $-0.99(-1 \cdot 77,-0 \cdot 22)^{*}$ & $-1 \cdot 80(-3 \cdot 10,-0 \cdot 49)^{* *}$ & $-1.66(-2.61,-0.72)^{* *}$ \\
\hline \multicolumn{5}{|l|}{ Model 2} \\
\hline High intake $^{a}$ & - & - & - & - \\
\hline Moderate intake & $-0 \cdot 11(-0 \cdot 80,0 \cdot 59)$ & $-0 \cdot 23(-0 \cdot 77,0 \cdot 31)$ & $-0 \cdot 46(-1 \cdot 50,0 \cdot 58)$ & $-0.91(-1 \cdot 82,0 \cdot 006)$ \\
\hline Low intake & $-0 \cdot 15(-0.85,0 \cdot 54)$ & $-0 \cdot 70(-1 \cdot 23,-0 \cdot 16)^{*}$ & $-1.50(-2 \cdot 54,-0 \cdot 46)^{* *}$ & $-1 \cdot 52(-2 \cdot 44,-00 \cdot 61)^{* *}$ \\
\hline \multicolumn{5}{|l|}{ Model 3} \\
\hline High intake ${ }^{a}$ & - & - & - & - \\
\hline Moderate intake & $-0.04(-0.74,0.66)$ & $-0.09(-0.62,0.45)$ & $-0 \cdot 34(-1 \cdot 38,0 \cdot 70)$ & $-0.73(-1.63,0.18)$ \\
\hline Low intake & $-0.04(-0 \cdot 74,0 \cdot 66)$ & $-0.40(-0.94,0.15)$ & $-1 \cdot 27(-2 \cdot 31,-0 \cdot 22) *$ & $-1.07(-1.99,-0 \cdot 15)^{*}$ \\
\hline
\end{tabular}

${ }^{a}$ Reference category. ${ }^{*} p<0 \cdot 05,{ }^{* *} p<0 \cdot 01$ compared to reference category. For boys, low FV intake was defined as $<135 \cdot 09 \mathrm{~g} / \mathrm{d}$, moderate $\mathrm{FV}$ intake as $135 \cdot 09-237 \cdot 71$ $\mathrm{g} / \mathrm{d}$, high FV intake as $>237 \cdot 71 \mathrm{~g} / \mathrm{d}$. For girls, low FV intake was defined as $<147 \cdot 43 \mathrm{~g} / \mathrm{d}$, moderate FV intake as $147 \cdot 43-267 \cdot 57 \mathrm{~g} / \mathrm{d}$, high FV intake as $>267 \cdot 57 \mathrm{~g} / \mathrm{d}$. Model 1: unadjusted; Model 2: adjusted for age, height, weight, pubertal status, energy intake; Model 3: adjusted for age, height, weight, pubertal status, energy intake, physical activity

Increased FV consumption in adolescence is associated with increased vertical jump power (muscle power). An association between increased FV consumption and grip strength (muscle strength) in girls was also evident, however, this became non-significant when adjusted for physical activity. Intervention studies are required to determine whether grip strength (muscle strength) and vertical jump power (muscle power) can be improved through increased FV consumption.

1. Semba RD, Blaum C, Guralnik JM, et al. (2003) Aging ClinExp Res 15, 482-7.

2. Semba RD, Lauretani F, Ferrucci L (2007) Arch BiochemBiophys 15, 141-145.

3. Cesari M, Penninx BW, Pahor M, et al. (2004) Am J ClinNutr 59, 242-8.

4. Robinson SM, Jameson KA, Batelaan SF, et al. (2008) J Am GeriatrSoc 56, 84-90. 